\title{
Efeito de diferentes densidades populacionais e do sexo sobre o desempenho e uniformidade em frangos de corte
}

Ricardo de ALBUQUERQUE ${ }^{1}$ Luciana Karina MARCHETTI $^{1}$ Antonio César Alves FAGUNDES 1

Letícia Cardoso

BITTENCOURT ${ }^{1}$

Messias Alves da TRINDADE NETO $^{1}$

Felix Ribeiro de LIMA $^{1}$

\section{Correspondência para:}

RICARDODEALBUQUERQUE

Departamento de Nutrição e Produção

Animal

Faculdade de Medicina Veterinária e

Zootecnia

Universidade de São Paulo

Av. Duque de Caxias Norte, 225

13635-900 - Pirassununga - SP

ricalbuq@usp.br

Recebido para publicação: 09/08/2004 Aprovado para publicação: 01/06/2005

1 - Departamento de Nutrição e Produção Animal da Faculdade de Medicina Veterinária e Zootecnia da Universidade de São Paulo, Pirassununga - SP

\section{Resumo}

O experimento teve a finalidade de comparar o desempenho e uniformidade de frangos de corte separados por sexo e criados em diferentes densidades populacionais. Para tanto foram utilizados 1800 pintos de 01 dia da linhagem Ross, distribuídos em 36 parcelas experimentais (Boxes), de acordo com um delineamento experimental tipo blocos ao acaso, obedecendo um modelo fatorial 3x2 com 3 sexos (Machos, Fêmeas e Misto) e duas densidades populacionais (10 e 15 aves $/ \mathrm{m}^{2}$ ), com 6 repetições por tratamento, sendo 40 ou 60 aves por repetição. As rações experimentais foram formuladas à base de milho e farelo de soja. Foram efetuadas medidas semanais de consumo de ração e peso dos animais, e ao final de 45 dias os animais foram abatidos. Ao final do período experimental, os resultados mostraram que os maiores valores de peso, consumo, Conversão Alimentar e Ganho Médio Diário foram verificados na menor taxa de lotação empregada, havendo significância estatística $(\mathrm{p}<0,05)$ para os itens : consumo (todos os períodos) e Conversão Alimentar (1-21 e 22-45 dias). Todavia, o incremento na taxa de lotação apresentou maiores viabilidade e uniformidade.Quanto aos sexos empregados, foram observados melhores valores para os machos, havendo diferença significativa $(p<0,05)$ para peso corporal (21 e 45 dias), consumo (22-45 e 1-45 dias), Conversão Alimentar (1-45 dias) e Ganho Médio Diário.

\section{Introdução}

O dinamismo da produção avícola, determinado pelo ciclo criatório curto e pelo sistema intensivo de criação, exige cada vez mais, a otimização do uso dos recursos envolvidos no processo. Atualmente, diversos criadores estão aumentando as taxas de lotação dos aviários, na busca de alternativas para viabilizar economicamente a criação, visto que o aumento da densidade populacional permite produzir maior quantidade de carne por unidade de área ${ }^{1}$.

Produções superiores a $30 \mathrm{Kg}$ de carne por metro quadrado são consideradas criações em alta densidade e o objetivo deste tipo de criação é a redução dos custos ${ }^{2}$.
Todavia, tem-se verificado uma redução no peso ao abate de aves mantidas sob maiores lotações ${ }^{3}$, e também um incremento nos índices de mortalidade e de condenação de carcaça ${ }^{4}$. As tendências atuais dos sistemas intensivos de criação ressaltam a importância do bem estar animal. Hurnick ${ }^{5}$, seguindo essa tendência, relata que as altas densidades prejudicam as aves, levando a resultados insatisfatórios e qualidade de carcaça inferior. O desenvolvimento tecnológico das condições de criação faz-se, portanto, indispensável para a melhoria do ambiente onde são produzidas as aves.

Além disso, fatores como uniformidade e viabilidade dos lotes são altamente influenciados pelo sexo das aves, pois fêmeas 
e machos têm necessidades diferentes, e os últimos necessitam de maior quantidade de alimento $^{6}$. De acordo com Silva, Garcia e Mendes ${ }^{7}$, a uniformidade dos lotes é um fator que pode influenciar no rendimento e na qualidade de carcaça. Segundo Tabajara ${ }^{8}$, a popularização das linhagens auto-sexáveis evidenciou um grande diferencial de peso vivo entre os frangos, principalmente a partir da metade final do ciclo criatório. Este diferencial é correlacionado ao sexo, sendo que, machos criados sob as mesmas condições que fêmeas, apresentam pesos corporais significativamente maiores. $\mathrm{O}$ manejo da criação em sexos separados proporciona uma melhor regulagem dos equipamentos, e desta forma é possível obter-se uma melhor uniformidade no consumo destas aves.

Diante do exposto, realizou-se o presente trabalho com o objetivo de verificar o efeito da taxa de lotação e do sexo das aves sobre os parâmetros zootécnicos de interesse econômico: desempenho e uniformidade, em frangos de corte criados até 45 dias de idade.

\section{Materiais e Métodos}

O experimento foi conduzido no galpão experimental de frangos de corte da Faculdade de Medicina Veterinária e Zootecnia da Universidade de São Paulo, Campus de Pirassununga, SP. O clima da região é do tipo Cwan de Köppen, ou seja, subtropical, com inverno seco e verão quente e chuvoso?. Foram utilizadas 1800 aves da linhagem comercial Ross, sendo 900 machos e 900 fêmeas, criadas em idênticas condições até 45 dias de idade, em 36 boxes com 1,70 m de frente por 2,50 $\mathrm{m}$ de lado. Em metade dos boxes utilizou-se a densidade de 10 aves $/ \mathrm{m}^{2}$ e na outra metade a densidade de 15 aves $/ \mathrm{m}^{2}$, e de acordo com os sexos, foram utilizados 12 boxes para cada um dos três grupos (macho, fêmea e misto). As aves foram criadas em piso coberto por cama de casca de amendoim, com espessura de $5 \mathrm{~cm}$.
Os tratamentos A e B foram compostos pelos machos nas densidades de 10 e 15 aves $/ \mathrm{m}^{2}$ respectivamente, os tratamentos C e D pelas fêmeas $(10$ e 15 aves $/ \mathrm{m}^{2}$ respectivamente), enquanto que os tratamentos $\mathrm{E}$ e $\mathrm{F}$ foram mistos ou compostos com $50 \%$ das aves de cada sexo, com as densidades respectivas de 10 e 15 aves $/ \mathrm{m}^{2}$. O manejo adotado e os equipamentos utilizados foram os convencionais para frangos de corte, e o programa alimentar seguiu as recomendações do National Research Council ${ }^{10}$. Na tabela 1 , encontram-se as fórmulas das rações utilizadas nos diferentes períodos experimentais e suas respectivas análises calculadas.

Para verificar o efeito do sexo e da densidade populacional sobre o desempenho foram analisados os valores médios de peso vivo (inicial, aos 21 dias e aos 45 dias), consumo de ração (períodos de 1-21, 22-45 e 1-45 dias), conversão alimentar (períodos de 1-21, 22-45 e 1-45 dias), ganho médio diário (GMD), calculado através do peso final dividido pelo número de dias do experimento, e $\mathrm{Kg}$ de frangos produzidos por $\mathrm{m}^{2}$ de área, mediante divisão do peso total das aves vivas pela área usada na sua criação. A viabilidade (expressa em percentagem de aves vivas), e a uniformidade (considerando-se aves uniformes aquelas dentro da faixa de variação de $10 \%$ do peso médio para mais ou para menos), foram avaliadas no final do experimento.

A análise experimental foi feita em delineamento inteiramente casualizado, em arranjo fatorial do tipo $2 \times 3$, com os fatores: densidade (10 e 15 aves $/ \mathrm{m}^{2}$ ) e sexo (macho, fêmea e misto), com seis tratamentos (grupos) e cinco repetições. Foi procedida análise de variância e as médias comparadas pelo teste de Tukey ${ }^{11}$.

\section{Resultados e Discussão}

Os valores médios obtidos para o desempenho das aves nas diferentes densidades são apresentados na tabela 2. Os 
Tabela 1-Composição percentual e resultado da análise calculada das rações nas diferentes fases do experimento. Pirassununga, SP, 2003

\begin{tabular}{|c|c|c|c|}
\hline & \multicolumn{2}{|c|}{ Fórmula (\%) } & \multirow[b]{2}{*}{$\begin{array}{l}\text { Fase fina } \\
\text { (43-45 dias) }\end{array}$} \\
\hline Ingredientes & $\begin{array}{l}\text { Fase inicial } \\
\text { (1-21 dias) }\end{array}$ & $\begin{array}{l}\text { Fase de crescimento } \\
\text { (22-42 dias) }\end{array}$ & \\
\hline Milho & 63,70 & 52,26 & 57,11 \\
\hline F. Soja & 28,00 & 40,13 & 34,00 \\
\hline Óleo de Soja & 4,50 & 3,52 & 4,90 \\
\hline Sal & 0,35 & 0,35 & 0,35 \\
\hline Calcário (38) & 1,24 & 1,60 & 1,60 \\
\hline Fosfato Bicálcico & 1,60 & 1,14 & 0,95 \\
\hline Suplemento Min. e Vit.* & 0,40 & 0,40 & 0,40 \\
\hline Metionina & 0,50 & 0,50 & 0,50 \\
\hline E. Metabolizável (kcal/kg) & 2950 & 3100 & 3150 \\
\hline Proteína Bruta (\%) & 22,5 & 20,0 & 18,0 \\
\hline Metionina (\%) & 0,47 & 0,35 & 0,30 \\
\hline Metionina + Cistina $(\%)$ & 0,71 & 0,65 & 0,60 \\
\hline Cálcio (\%) & 0,95 & 0,95 & 0,90 \\
\hline Fósforo Disponível (\%) & 0,45 & 0,35 & 0,30 \\
\hline
\end{tabular}

*Fornecimento por kg de produto:

Fase Inicial: vitamina A 1500000 UI, vitamina D 200000 UI, vitamina E 2000 mg, vitamina K 490 mg, vitamina B 660 mg, vitamina B 2000 mcg , niacina $1000 \mathrm{mg}$, colina $25000 \mathrm{mg}$, pantotenato de cálcio $1000 \mathrm{mg}$, ferro $50000 \mathrm{mg}$, cobre $7500 \mathrm{mg}$, manganês $12000 \mathrm{mg}$, cobalto $21 \mathrm{mg}$, iodo $125 \mathrm{mg}$, zinco $10000 \mathrm{mg}$, selênio $25 \mathrm{mg}$, coccidiostático $250000 \mathrm{mg}$, promotor de crescimento $2500 \mathrm{mg}$, antioxidante 1280 $\mathrm{mg}$, veículo qsp $1000 \mathrm{~g}$

maiores valores de peso, consumo, CA e GMD foram verificados na menor taxa de lotação empregada, havendo significância estatística $(\mathrm{p}<0,05)$, em todos os períodos estudados, para os itens consumo e CA. Na tabela 3 apresenta-se os valores médios de desempenho para os sexos empregados e foram verificados os melhores valores para o grupo de machos criados separadamente. Foi observada diferença significativa $(p<0,05)$ para os itens peso corporal (aos 21 e aos 45 dias), consumo de ração (períodos de 22 a 45 e 1 a 45 dias), CA (período de 1 a 45 dias) e GMD.

Os valores médios de desempenho para a interação dos fatores sexo e densidade são apresentados na tabela 4, onde é possível observar que houve interação significativa entre as fontes de variação (sexo e densidade) para os itens: peso corporal aos 21 e 45 dias $(p<0,01)$, consumo de ração do $1^{\circ}$ ao $21^{\circ}$ dia de idade $(\mathrm{p}<0,01)$ e do $1^{\circ}$ ao $45^{\circ}$ e $22^{\circ}$ ao $45^{\circ}$ dias $(\mathrm{p}<0,05)$, conversão alimentar de 1-21 dias ( $<<0,05)$, GMD $(\mathrm{p}<0,05)$. A produção de $\mathrm{Kg}$ de frango por metro quadrado de galpão nos diferentes tratamentos do experimento é também apresentada, e foi verificada interação significativa $(p<0,05)$, bem como a superioridade dos machos na maior densidade empregada. Quanto aos valores médios obtidos para viabilidade e uniformidade, obteve-se que a maior taxa de lotação (15 aves/m2) apresentou melhores índices de viabilidade e de uniformidade para o período total de criação $(p<0,01)$ enquanto que entre os grupos separados por sexo não houveram diferenças significativas.

A interação densidade $\mathrm{x}$ sexo revelou os melhores índices de viabilidade para o período total da criação $(\mathrm{p}<0,01)$ no grupo $\mathrm{B}$, e a maior uniformidade, conforme a tabela 4, ocorreu nos grupos com aves do sexo feminino (C e D). No período inicial da criação (1-21 dias), não houve interação entre os fatores sexo e densidade para a viabilidade, sendo que permaneceu estatisticamente igual em ambas densidades e nos diferentes grupos separados por sexo. O consumo de ração foi maior na densidade de 10 aves $/ \mathrm{m}^{2}$ em todos os períodos da 
Tabela 2-Valores médios de desempenho das aves nas diferentes densidades populacionais estudadas. Pirassununga, SP, 2003

Densidade (Aves $/ \mathrm{r}^{2}$ )

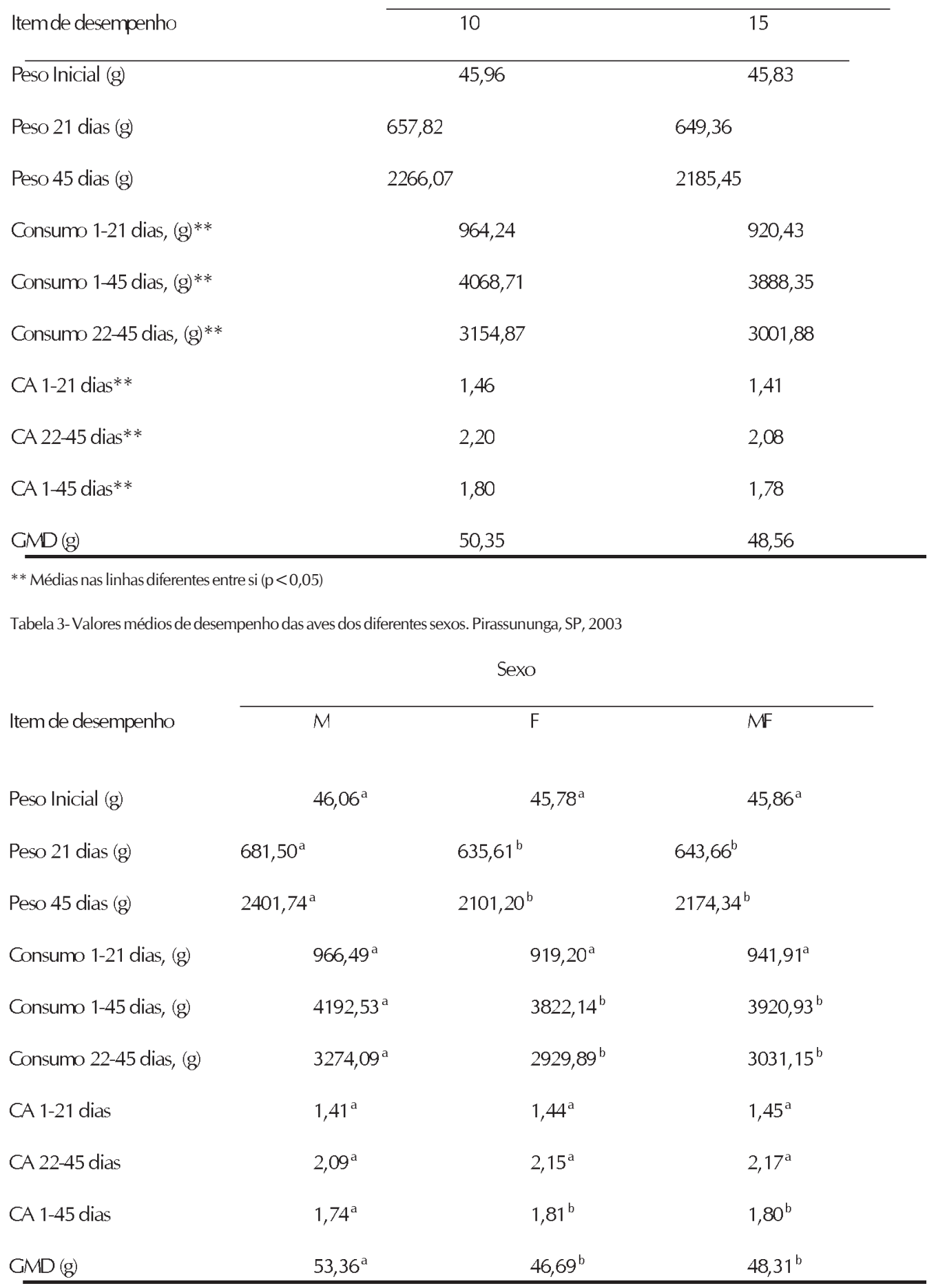

** Médias seguidas de letras diferentes nas linhas são diferentes entre si $(p<0,05)$ pelo Teste de Tukey 
Tabela4-Valores médios dedesempenhoedeuniformidadedas aves dos diferentes sexos nas densidades populacionais estudadas. Pirassununga, SP, 2003

\begin{tabular}{|c|c|c|c|c|c|c|}
\hline \multirow[b]{3}{*}{ Item de Desempenho } & \multicolumn{6}{|c|}{ Densidade $\left(\right.$ aves $/ \mathrm{m}^{2}$ ) / Sexo } \\
\hline & \multicolumn{3}{|c|}{10} & \multicolumn{3}{|c|}{15} \\
\hline & $M$ & $F$ & $M F$ & $\bar{M}$ & $F$ & $\mathrm{MF}$ \\
\hline Peso inicial, g. & 46,30 & 45,80 & 45,80 & 45,82 & 45,76 & 45,93 \\
\hline Peso 21 d., g.* & 684,07 & 640,13 & 649,29 & 678,94 & 631,10 & 638,03 \\
\hline Peso 45 d., g. * & 2448,56 & 2141,13 & 2208,51 & 2354,91 & 2061,27 & 2140,17 \\
\hline Consumo 1-21 d, g.* & 983,21 & 931,60 & 977,90 & 949,77 & 906,80 & 905,22 \\
\hline Consumo $1-45$ d., g.** & 4311,12 & 3917,61 & 3977,41 & 4073,94 & 3726,67 & 3864,45 \\
\hline Consumo $22-45 \mathrm{~d}, \mathrm{~g}^{* *}$ & 3384,30 & 3018,84 & 3061,47 & 3163,88 & 2840,94 & 3000,83 \\
\hline CA $1-21$ d.** & 1,43 & 1,45 & 1,50 & 1,39 & 1,43 & 1,41 \\
\hline CA $22-45 \mathrm{~d}$. & 2,18 & 2,22 & 2,20 & 2,00 & 2,09 & 2,15 \\
\hline CA $1-45 \mathrm{~d}$. & 1,77 & 1,83 & 1,80 & 1,73 & 1,80 & 1,80 \\
\hline GMD, $g^{*}$ & 54,41 & 47,58 & 49,07 & 52,32 & 45,80 & 47,55 \\
\hline Frango $/ \mathrm{m}^{2}, \mathrm{Kg} * *$ & 21,29 & 19,30 & 18,75 & 32,60 & 29,05 & 29,41 \\
\hline Viabilidade 1_21 d, \% & 98,50 & 99,45 & 99,00 & 98,28 & 99,00 & 99,33 \\
\hline Viabilidade 1_45d, \%* & 88,29 & 94,94 & 86,37 & 94,98 & 94,94 & 92,27 \\
\hline Unifomidade, \%* & 87,00 & 94,00 & 85,50 & 93,28 & 94,00 & 91,66 \\
\hline
\end{tabular}

*Interação significativa entre as fontes de variação $(p<0,01)$.

criação, e a conversão alimentar foi melhor também na menor taxa de lotação no período de 1-21 dias e no período de 2245 dias de idade. Estes resultados corroboram com Beg, Hawid e Ahmed ${ }^{12}$, que criando os frangos até 8 semanas de idade, encontraram expressivas diferenças entre o consumo e a conversão alimentar comparando o desempenho das aves nas densidades de 1000, 1500 e $2000 \mathrm{~cm}^{2}$ /ave. Luchesi ${ }^{1}$ relatou que em altas densidades, as aves apresentam dificuldade de acesso ao 
comedouro e maior gasto energético para se alimentar, explicando a queda no consumo e exigindo o emprego de rações com maior concentração de nutrientes.

O grupo de frangos machos criados separadamente apresentou maior peso corporal aos 21 e aos 45 dias, bem como maior GMD quando comparado ao grupo de fêmeas e ao grupo misto, o que já foi descrito por Han e Baker ${ }^{6}$, que relataram a maior rapidez no desenvolvimento dos machos e por Tabajara ${ }^{8}$, que afirmaram que machos criados sob as mesmas condições que fêmeas, apresentaram pesos corporais significativamente maiores. Foi verificada interação entre os fatores densidade e sexo, e com o grupo A obteve-se melhor GMD e maior consumo, sendo que os machos sempre atingiram peso de abate maior e apresentaram melhor conversão alimentar na fase inicial da criação. Por outro lado, Goldfus et al. ${ }^{3}$, encontraram interação entre os fatores densidade e sexo apenas para o item ganho de peso, e relataram ainda que a redução deste parâmetro nos frangos criados sob maiores taxas de lotação deve-se ao menor consumo. $\mathrm{O}$ aumento na densidade populacional atuou de forma negativa sobre o ganho de peso, como já foi observado por Elwinger ${ }^{13}$ que afirmou que frangos criados em densidades mais altas são em média, 27g. mais leves que os criados em densidades mais baixas. Assim, de acordo com o relatado por vários autores ${ }^{6,8,13,14}$, os resultados referentes ao peso vivo das aves obtidos no presente experimento, já eram esperados. Foram obtidos maiores valores para as aves do sexo masculino, em relação às com sexos combinados e às do sexo feminino, e o efeito negativo do aumento da densidade sobre o desempenho dos frangos, também já previsto, foi confirmado. Todavia, contrariando o exposto por Han e Baker ${ }^{6}$ e Silva, Garcia e Mendes ${ }^{6}$ no presente trabalho não foi observada diferença entre os sexos com relação à uniformidade e viabilidade, enquanto que entre as densidades, observou-se melhor uniformidade e melhor viabilidade (período de 1-45 dias) para a maior taxa de lotação, também ao contrário do descrito por vários autores $^{1,7,9,16}$. Apesar de expressivas perdas em alguns itens de desempenho, e das piores condições observadas no material da cama, os valores de $\mathrm{Kg}$ de frango obtidos por metro quadrado de área, revelam que o incremento na densidade e a sexagem das aves é viável. Esses dados eram esperados, estando de acordo $\operatorname{com}^{4,9,16}$.

\section{Conclusões}

Nas condições em que o presente trabalho foi conduzido, é possível concluir-se que

1 - Com relação ao sexo, os machos apresentaram melhor desempenho;

2 - O incremento na taxa de lotação atuou de forma negativa sobre o peso médio diário, todavia apresentou maiores viabilidade e uniformidade;

3 - Houve interação entre os fatores sexo e densidade para todas as fontes de variação;

4 - Apesar da maior densidade ter acarretado perda nos itens de desempenho, foi possível produzir mais $\mathrm{Kg}$ de frango por metro quadrado de área.

\section{Effect of different stocking rates and sex on the performance and uniformity of broilers}

\section{Abstract}

This experiment was developed to compare the performance and uniformity of broilers chickens as related to sex and different stocking rates. 1800 one day Ross breed chicks were distributed in 36 boxes, on a completely randomized block design, $3 \times 2$ factorial, with 3 sex (male, female and mixed) and 2 stocking $\left(10\right.$ and $\left.15 \mathrm{birds} / \mathrm{m}^{2}\right)$. There
Key-words: Broiler chicken. Performance. Stocking. Uniformity. 
were six replications per treatment, with 40 or 60 birds per replication. The experimental ration was formulated with corn and soy bean meal. The results were taken weekly, and after 45 days the animals were slaughtered. The results showed an increase in weight, consumption food, food conversion and daily weight gain in the smaller stocking, with statistical significance $(p<0,05)$ for: food consumption (all period) and food conversion (1-21 and 22-45 days). However, the increase in stocking showed better values in viability and uniformity. With regard the sex, better values were seen for males, with statistical significance $(\mathrm{p}<0,05)$ for weight (21 and 45 days), food consumption(22-45 and 1-45 days), food conversion (1-45 days) and daily weight gain.

\section{Referências}

1 LUCHESI, J. B. Custo-benefício da criação de frangos de corte em alta densidade no inverno e no verão. In: CONFERÊNCIA APINCO DE CIÊNCIA E TECNOLOGIA AVÍCOLAS, 1998, Campinas. Anais... Campinas: Facta, 1998. p. 241-248.

2 SANTIN, A. R. Criação de frangos de corte com alta densidade. In: CONFERÊNCIA APINCO DE CIÊNCIA E TECNOLOGIA AVÍCOLAS, 1996, Curitiba. Anais..., Campinas: Facta, 1996. p. 119-32.

3 GOLDFLUS, F.; et al. Melhoria da produtividade em frangos de corte com aumento da densidade populacional na estação fria do ano. In: CONFERÊNCIA APINCO DE CIÊNCIA E TECNOLOGIA AVÍCOLAS, 1994, Campinas. Trabalhos de pesquisa... Campinas: Facta, 1994. p.111-112.

4 SIMON, V. A. Aspectos sanitários de criações em altas densidades. In: SIMPÓSIO SOBRE AMBIÊNCIA, SANIDADE E QUALIADADE DE CARCAÇA DE FRANGOS DE CORTE, 1997, Concórdia. Anais... Concórdia: EMBRAPA-CNPSA, 1997. p. 14-18.

5 HURNIK, J. F. Poultry welfare. In: HUNTON, P. Poultry production. Amsterdam: Elsevier, 1995. p. 561578.

6 HAN, Y.; BAKER, D. H. Effects of sex, heat stress, body weight, and genetic strain of the dietary lysine requeriment of broiler chicks. Poultry Science, v. 72, n. 4, p. 701-708, 1993.

7 SILVA, A. B. P.; GARCIA, E. A.; MENDES, A. A. Efeito do sexo sobre desempenho e uniformidade em lotes de frangos de corte. In: CONFERÊNCIA APINCO DE CIÊNCIA E TECNOLOGIA AVÍCOLAS, 1994, Campinas. Trabalhos de pesquisa. Campinas: Facta, 1994. p. 107-108.

8 TABAJARA, P. Criação de frangos de corte sexados: vantagens $x$ desvantagens e programas de alimentação. In: CONFERÊNCIA APINCO DE CIÊNCIA E TECNOLOGIA AVÍCOLAS, 1997, Campinas. Anais... Campinas: Facta, 1997. p. 259-283.

9 OLIVEIRA, J. B.; PRADO, H. Levantamento pedológico do Estado de São Paulo: Quadricula de São C. Memorial Descritivo. Boletim Técnico do Instituto Agronômico de Campinas, v. 98, p. 187-195, 1994.

11 STEEL, R. G.; TORRIE, S. H. Principles and procedures of statistics with special reference to the biological sciences. New York: McGraw-Hill, 1991. $600 \mathrm{p}$.

12 BEG, M. A. H.; HAMID, M. A.; AHMED, S. The effect of stocking density on the performances of broiler chicks. Poultry Adviser, v. 28, n. 8, p. 41-46, 1995.

13 ELWINGER, K. Broiler production under varyingpopulation densities: a field study. Archiv fur Geflugelkunde, v. 59, n. 4, p. 209-215, 1995.

14 IMAEDA, N. Influence of the stocking density and rearing season on incidence of sudden death syndrome in broiler chickens. Poultry Science, v. 79, n. 2, p. 201204, 2000.

15 GRAÇAS, A. S.; et al. Densidade populacional de frangos de corte em diferentes épocas do ano. Revista da Sociedade Brasileira de Zootecnia, v. 19, n. 3, p. 186-96, 1990.

16 JONES, R.B. Fear and adaptability in poultry: insights, implications and imperatives. Wolrd's Poultry Science Journal, v. 52, n. 2, p. 131-174, 1996.

10 NATIONAL RESEARCH COUNCIL. Nutrient requirement poultry. 9. ed. Washington: National Academy Press, 1994. 156 p. 